\title{
Patient-reported and clinician-perceived barriers to group visits
}

\begin{abstract}
Background: Group visits are a healthcare delivery model with potential for improving disease-oriented outcomes and patient and clinician satisfaction, yet they are rarely implemented in routine practice. This study explored patient-reported and clinician-perceived barriers that may be modified to enhance group visit opportunities.

Methods: Adult patients with type 2 diabetes $(n=48)$ and primary care clinicians $(\mathrm{n}=107)$ were queried regarding barriers to attending and implementing diabetes group visits, respectively. Chi-square tests were used to compare patient-reported and clinician-perceived barriers to group visit attendance. Descriptive statistics identified optimal time of day for group visits.
\end{abstract}

Results: Clinicians were more likely to identify logistical barriers for patients (transportation, work, copays); patients were more likely to feel that they did not need additional diabetes management support. Both patients and clinicians named evenings as the optimal time of day for group visits, followed by afternoons.

Conclusion: There were consistent differences between patient-reported and clinicianperceived barriers to attending diabetes group visits. Further exploration of why patients feel they do not need additional support may provide insight into recruitment challenges for group visits and other self-management support modalities.
Volume 5 Issue I - 2017

\section{Beth Careyva, Melanie Johnson, Kyle Shaak BS, Elaine Seaton Banerjee, Liselly Diaz, Brian Stello}

Lehigh Valley Health Network, USA

Correspondence: Beth A Careyva, Department of Family Medicine, Lehigh Valley Health Network, Allentown, Pennsylvania, USA, Tel 4848623059, Fax 4848623096, Email beth_a.careyva@lvhn.org

Received: October 10, 2016 | Published: January 26, 2017

\section{Background}

Diabetes group visits include individualized consultation followed by group self-management education. ${ }^{1}$ Group visits have been found to improve diabetes-related biomarkers in many settings and populations. ${ }^{2,3}$ Despite this, both patients and clinicians experience barriers to the successful implementation of group visits in routine practice. Prior studies demonstrated patient interest in diabetes education and peer support. ${ }^{4,5}$ The purpose of this study was to evaluate patient-reported and clinician-perceived barriers for group visits and to use this information to identify strategies to promote successful group visit implementation. We hypothesized that patients and clinicians would be in agreement regarding the barriers for patient attendance. We also sought to identify the optimal time of day for group visits, with a hypothesis that patients prefer evening group visits, whereas clinicians prefer morning or afternoon group visits.

\section{Methods}

A registry of adult patients with type 2 diabetes was created in one primary care practice with a diverse patient population, in which $25.0 \%$ of patients had Medicaid and $15.5 \%$ had Medicare insurance. Because a validated tool was not available, the study team created a 10-item patient survey. This survey was piloted in a sample of 50 patients. A revised version was mailed to 187 patients; 48 patients completed the survey $(25.7 \%$ response rate). Similarly, a 10 -item survey was mailed to 246 primary care clinicians within a regional practice-based research network (PBRN) to explore experiences with implementation of diabetes group visits, with a response rate of
43.5\%. Detailed methods for the patient and clinician surveys may be found in previously published papers. ${ }^{4,6}$ Chi-square tests were used to compare patient-reported and clinician-perceived barriers to diabetes group visits (transportation, work and/or other responsibilities, copays, prefer to not discuss diabetes in a group setting, and do not need additional support). These checked or un-checked responses were converted to dichotomous variables for chi-square analysis. Descriptive statistics were used to assess patient and clinician preferences for time of day for group visits (morning, afternoon, evening).

\section{Results}

This study included 48 adult patients with type 2 diabetes and 107 primary care clinicians. Detailed demographic information for both groups has been previously reported. ${ }^{4,6}$ Dichotomous "yes" or "no" responses to the following survey questions are reported in Table 1) What factors do you think may make it difficult for your patients to attend group visits? 2) Which of the following factors might prevent you from attending a diabetes group visit? Clinicians were significantly more likely to perceive transportation, work, and privacy concerns as barriers; patients were significantly more likely to report "do not need additional support" as a barrier to attending diabetes group visits. Patients and clinicians were also asked about preferred time of day for group visits. Patients reported preference for time of group visits as follows: $6.9 \%$ morning, $24.1 \%$ afternoon, $69 \%$ evening. Clinicians' selected preferences included $29.6 \%$ morning, $33.3 \%$ afternoon, and $37.0 \%$ evening. 
Table I Patient-reported and clinician-perceived barriers to attending diabetes group visits

\begin{tabular}{|c|c|c|c|c|c|c|}
\hline & \multicolumn{6}{|c|}{ Number identifying as a barrier } \\
\hline & \multicolumn{2}{|c|}{ Clinician $(n=105)$} & \multicolumn{2}{|c|}{ Patient $(n=48)$} & \multirow{2}{*}{$\mathbf{X}^{2}$} & \multirow{2}{*}{ p-value } \\
\hline & Yes & No & Yes & No & & \\
\hline Transportation & 45 & 60 & 6 & 41 & 13.186 & $<.001$ \\
\hline Work and/or other responsibilities & 73 & 32 & 17 & 31 & $|5.82|$ & $<.001$ \\
\hline Difficulty in affording copy & 28 & 77 & 8 & 40 & 1.831 & 0.176 \\
\hline Prefer not to discuss health in a group setting & 70 & 35 & 10 & 38 & 27.738 & $<.001$ \\
\hline Do not need additional support & 4 & 101 & 8 & 40 & 7.534 & 0.006 \\
\hline
\end{tabular}

*Survey responses for this question were available for 105 of 107 clinician respondents

**Patient and clinician participants were able to select multiple responses.

\section{Discussion}

Patient-reported and clinician-perceived barriers were significantly different; clinicians were more likely than patients to cite logistical barriers including transportation and work and/or other responsibilities. While some may experience barriers, group visits are still accessible for many patients to promote patient engagement and foster adherence to treatment regimens. Inaccurate clinician assumptions regarding patient barriers may negatively impact the likelihood of implementing group visits in practice. In addition to logistical barriers, significantly more patients reported "do not need additional support" as compared to clinicians. This discrepancy suggests a disconnect between patients and clinicians in appraisal of need for additional diabetes support and is consistent with prior research, in which clinicians were more likely than patients to view diabetes as "serious."7Althoughpatientswhose diabetes is well-controlled may not need current additional support; patients' understanding of the progressive nature of diabetes should be explored. Further qualitative data is needed to investigate how patients understand diabetes-related prognosis and how their perceptions may impact motivation to enroll in group visits. Lastly, physicians were significantly more likely to name "prefer not to discuss health in a group setting" as a barrier than were patients. Patients may find group visits to be a safe space to share concerns and gain support from peers. ${ }^{7,8}$ Another common barrier is time of day when group visits are offered. Both patients and clinicians indicated that evening visits were preferable. It was expected that patients with work and other responsibilities would be more available in evenings. However, a surprising outcome was that clinicians had the same preference, especially when considering the resources required to keep the office open. Clinicians may view the only feasible option for group visits to be an "add-on" to regularly scheduled appointments. This preference for evening hours also speaks to the current trend toward afterhours health care to increase access for patients who have difficulty accessing care within traditional hours. ${ }^{9}$ A limitation of this study includes the sample size. Patients were recruited from one primary care practice and clinicians were recruited from one PBRN. Given the number of patients within this practice with Medicaid or Medicare insurances, they may have been more likely to experience logistical challenges to group visit attendance. This may also have biased some clinicians to anticipate barriers based on socioeconomic status, though clinicians represented in the survey were from practices with varied demographics across the PBRN. Additionally, the survey did not query patients regarding challenges with insurance coverage, which may be included in future surveys. Further study of both patients and clinicians is needed to determine generalizability. An additional limitation was inclusion of survey data only. Further qualitative exploration is needed to promote dissemination of group visits in practice, particularly for responses that differed from hypotheses.

\section{Implications}

Logistical barriers for patients to attend diabetes group visits were perceived and reported by clinicians at a higher proportion than were reported by adult patients with type 2 diabetes. This clinician perception of barriers should not impede further incorporation of group visits into practice. Many patients did not feel that they needed additional support, which warrants further exploration. Additionally, offering group visits in evenings may be preferable for both patients and clinicians.

\section{Acknowledgements}

The authors gratefully acknowledge the Dorothy Rider Pool Health Care Trust for funding this work. In addition, they acknowledge the contributions of Jacqueline Grove in editing and manuscript preparation. Ms. Grove has no conflicts of interest to disclose.

\section{Conflict of interest}

The author declares no conflict of interest.

\section{References}

1. Riley SB, Marshall ES. Group Visits In Diabetes Care: A Systematic Review. The Diabetes Educator. 2010;36(6):936-944.

2. Caballero CA, Firek AF, Kashner TM. Effect of Group Medical Appointments On Glycemic Control Of Patients With Type 2 Diabetes. Diabetes Spectr. 2015;28(4):245-251.

3. Berry D, Williams W, Hall EG, et al. Imbedding Interdisciplinary Diabetes Group Visits Into A Community-Based Medical Setting. Diabetes Educ. 2016;42(1):96-107.

4. Careyva BA, Johnson MB, Shaak K, et al. Patient-Reported Barriers And Limitations To Attending Diabetes Group Visits. J Prim Care Community Health. 2015;6(4):279-281.

5. Gazmararian JA, Ziemer DC, Barnes C. Perception of Barriers to Self-Care Management among Diabetic Patients. Diabetes Educ. 2009;35(5):778-788.

6. Careyva BA, Johnson MB, Shaak K, et al. Clinician-Reported Barriers to Group Visit Implementation. J Prim Care Community Health. 2016;7(3):188-193. 
7. Clark M, Hampson SE. Comparison of Patients' And Healthcare Professionals' Beliefs About And Attitudes Towards Type 2 Diabetes. Diabet Med. 2003;20(2):152-154.

8. Wong ST, Lavoie JG, Browne AJ, et al. Patient Confidentiality within the Context of group Medical Visits: Is There Cause For concern? Health Expect. 2015;18(5):727-739.
9. Patwardhan A, Davis J, Murphy P, et al. After-Hours Access of Convenient Care Clinics And Cost Savings Associated With Avoidance Of Higher-Cost Sites Of Care. J Prim Care Community Health. 2012;3(4):243-245. 